\title{
Transformation of nitrogen during microalgae liquefaction in sub-/supercritical ethanol
}

Yang Peng ${ }^{a}$, Chuxuan Zhang ${ }^{a}$, Xun Gong, ${ }^{a}$, , Shuang Wang ${ }^{b}$, Huping Liu ${ }^{a}$, Henghui Li ${ }^{a}$, Luqiu Lin ${ }^{a}$, Yun $Y u^{a}$

a State Key Laboratory of Coal Combustion, School of Energy and Power Engineering, Huazhong University of Science and Technology, 1037 Luoyu Road, Wuhan 430074, PR China ${ }^{\mathrm{b}}$ School of Energy Power Engineering, Jiangsu University, 212013 Jiangsu, China

* Corresponding Authors. E-mails: gx@hust.edu.cn (X. Gong); Tel: +86-27-87542417-8301, Fax: $+86-27-87545526$

\section{Submitted to Energy \& Fuels}

for consideration of publication in the Special Issue of Recent Advances in Thermochemical Processing of Biomass and Solid Wastes in China 


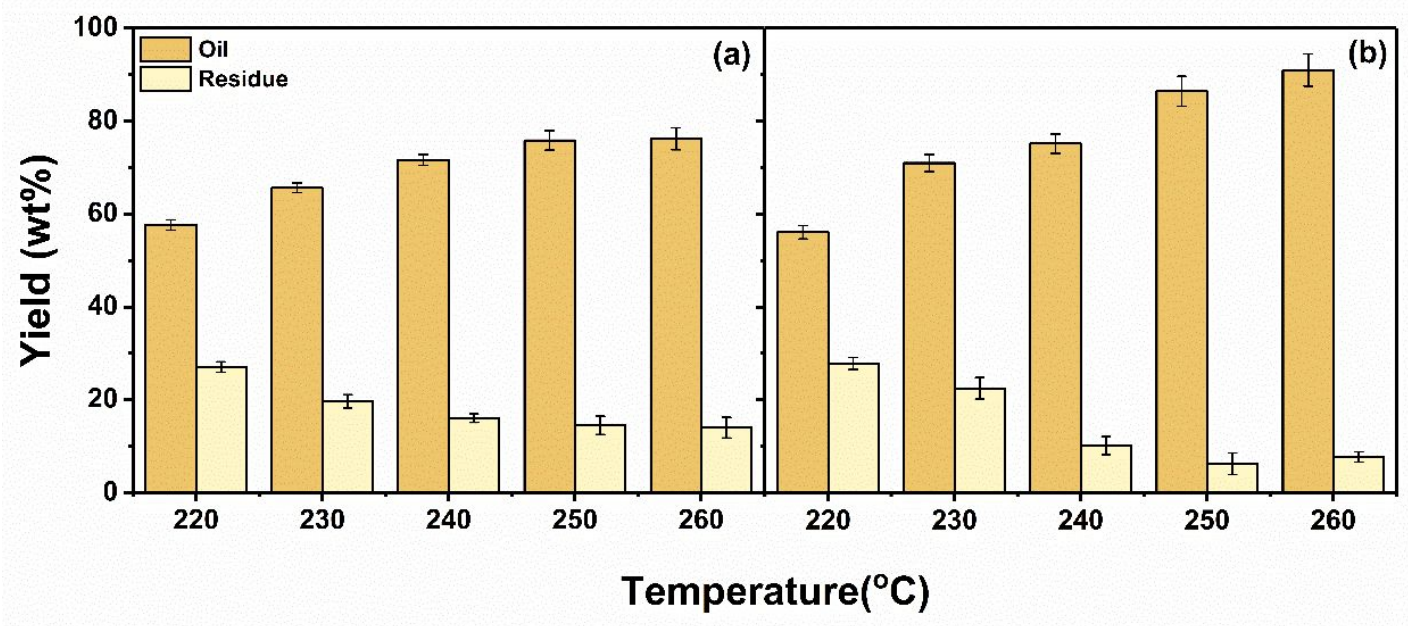

Figure S1. Effect of temperature on production yield of CV and SP. (a) CV and (b) SP. 


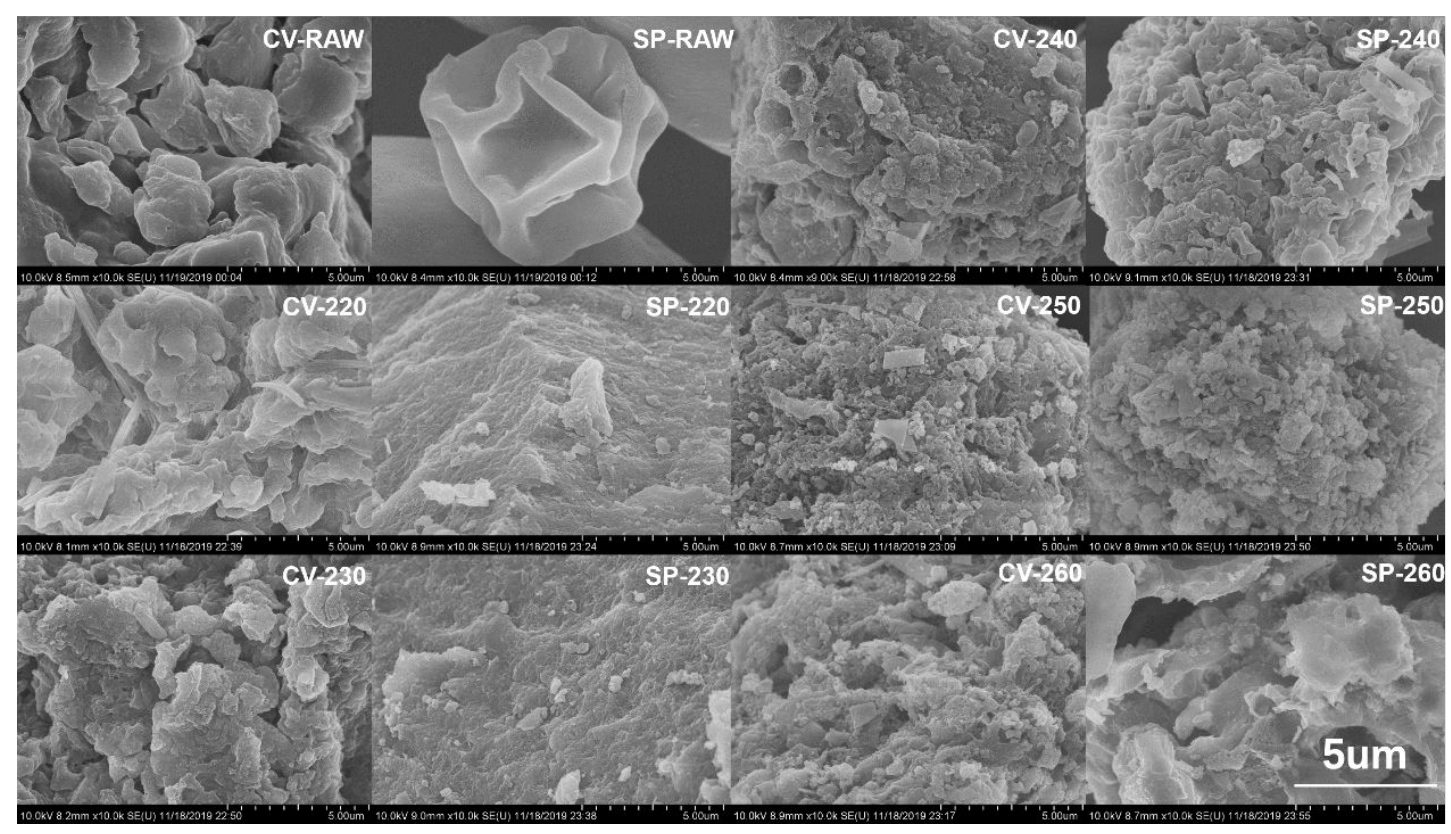

Figure S2. SEM images of raw feedstock and solid residue 
Table S1. Tentative identities and area $\%$ of major peaks (Relevancy $\geq 70 \%$ ) in oils produced from CV

\begin{tabular}{|c|c|c|c|c|c|}
\hline \multirow{2}{*}{ Compound name } & \multicolumn{5}{|c|}{ Area \% } \\
\hline & CV-220 & CV-230 & CV-240 & CV-250 & $C V-260$ \\
\hline Ethyl L(-)-lactate & 6.07 & 5.49 & 6.07 & 4.65 & 5.05 \\
\hline 2-Methylpyrazine & 1.81 & 2 & 2.12 & 2.07 & 2.28 \\
\hline Urethane & 1.48 & 1.31 & 1.29 & 0.88 & \\
\hline 2,5-Dimethyl pyrazine & 0.86 & 0.81 & 0.83 & 0.95 & 0.97 \\
\hline Ethylpyrazine & 0.46 & & & 0.38 & \\
\hline Pyrazine,2-ethyl-6-methyl- & 0.16 & & & & \\
\hline Ethyl levulinate & & & & 0.33 & \\
\hline 2,5-Dimethyl-3-ethylpyrazine & 0.21 & 0.52 & & 0.69 & \\
\hline Diethyl succinate & 2.23 & 2.31 & 2.47 & 2.28 & 3.73 \\
\hline 2-Pyrrolidinecarboxylic acid-5-oxo-, ethyl ester & 21.32 & 19.85 & 21.78 & 17.73 & 19.96 \\
\hline Aziridine, 2-methyl-3-(1-methylethyl)-, trans- & & 1.04 & & & \\
\hline Pyrrolo[1,2-a]pyrazine-1,4-dione,hexahydro- & 2.52 & 1.88 & 1.47 & 1.96 & 2.75 \\
\hline
\end{tabular}




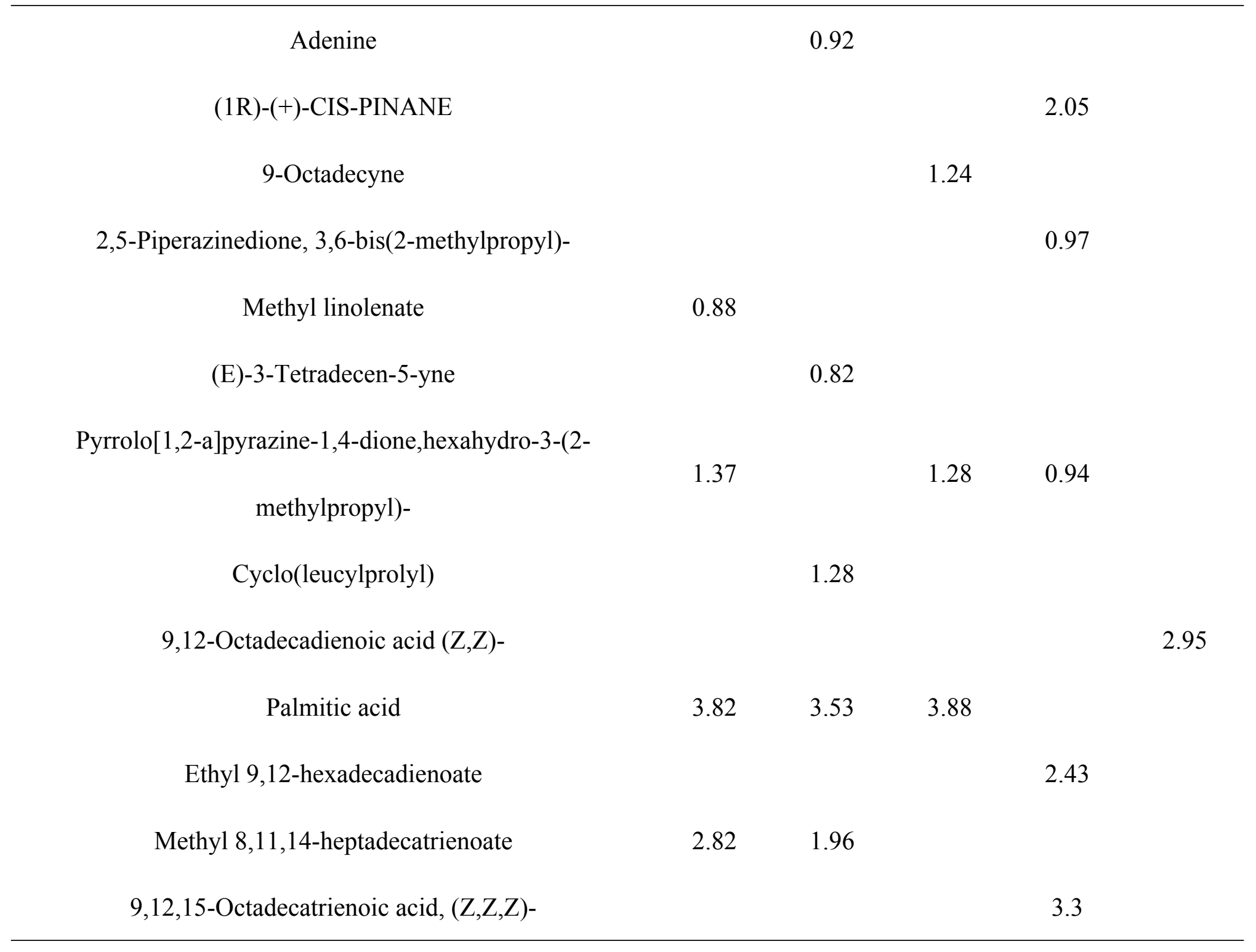




\begin{tabular}{|c|c|c|c|c|c|}
\hline 4-Amino-2-nitrophenol & & & & & 4.49 \\
\hline Pentanoic acid, 2-methyl-, anhydride & 1.48 & & & & \\
\hline 2,5-Dihydro-2,5-dimethoxyfuran & & 1.56 & & & \\
\hline hexanoic acid, anhydride & & & 1.52 & & \\
\hline Ethyl palmitate & 5.5 & 6.32 & 9.32 & & 12.52 \\
\hline Hexadecanoic acid, ethyl ester & & & & 8.48 & \\
\hline Ethyl 14-methyl-hexadecanoate & 0.74 & & & & \\
\hline Ethyl heptadecanoate & & & & 0.1 & \\
\hline Linoleic acid & 2.46 & 2.22 & 1.65 & 0.74 & \\
\hline Linolenic acid & 3.36 & 1.72 & 1.02 & & \\
\hline 9,12-Octadecadienoic acid (Z,Z)- & & & & 1.27 & \\
\hline 9,12-Octadecadienoic acid (Z,Z)-, ethyl ester & & & 10.36 & 9.53 & 1.04 \\
\hline Ethyl Linoleate & 6.85 & 7.95 & & & 13.39 \\
\hline (Z,Z,Z)-9,12,15-Octadecatrienoic acid, ethyl ester & 4.3 & 4.54 & 5.07 & 4.73 & \\
\hline
\end{tabular}




\begin{tabular}{|c|c|c|c|c|c|}
\hline lauramide & 1.74 & & & & \\
\hline Hexadecanamide & & 1.99 & & & \\
\hline Methyl 9,12-heptadecadienoate & 0.95 & & & & \\
\hline
\end{tabular}


Table S2. Tentative identities and area $\%$ of major peaks (Relevancy $\geq 70 \%$ ) in oils produced from SP

\begin{tabular}{|c|c|c|c|c|c|}
\hline \multirow{2}{*}{ Compound name } & \multicolumn{5}{|c|}{ Area \% } \\
\hline & SP-220 & SP-230 & SP-240 & SP-250 & SP-260 \\
\hline Ethyl lactate & 1.92 & 1.51 & 1.22 & 1.05 & 1.18 \\
\hline 2-Methylpyrazine & & & & 0.73 & 0.96 \\
\hline Urethane & 2.49 & 1.64 & 1.12 & 0.72 & \\
\hline Glycine, leucyl- (9CI) & & & & & 1.33 \\
\hline L-Leucine, ethyl ester & & & 0.49 & 0.57 & \\
\hline Diethyl succinate & & & & & 1.01 \\
\hline 2-Pyrrolidinecarboxylic acid-5-oxo-, ethyl ester & 18.72 & 15.32 & 14.64 & 14.78 & 17.6 \\
\hline Heptadecane & 8.71 & 7.6 & 5.32 & 5.01 & 4.39 \\
\hline Pyrrolo[1,2-a]pyrazine-1,4-dione,hexahydro- & 2.09 & 2.98 & 2.77 & & 3.22 \\
\hline Adenine & & 2.4 & 1.72 & 1.11 & \\
\hline Pinane & & & & 1.72 & \\
\hline
\end{tabular}




\begin{tabular}{|c|c|c|c|c|c|}
\hline 6-Octen-1-ol,3,7-dimethyl-, 1-formate & & 1.28 & 1.05 & 0.86 & \\
\hline Palmitic acid & 2.81 & 2.2 & & & \\
\hline 9-Hexadecenoic acid,ethyl ester & 4.24 & 3.8 & 4.03 & 4.1 & 4.75 \\
\hline Ethyl palmitate & 25.12 & 22.69 & 21.85 & 23.33 & 24.75 \\
\hline 2-Butyl-1-methylpyrrolidine & & & & 1.49 & \\
\hline Piperidine, 2-pentyl- & & 1.05 & 1.69 & & \\
\hline 8,11,14-Eicosatrienoicacid, $(8 \mathrm{Z}, 11 \mathrm{Z}, 14 \mathrm{Z})$ - & 8.36 & 7.41 & & & 1.44 \\
\hline cis-5,8,11-Eicosatrienoic acid, methyl ester & & & 7.49 & & 7.76 \\
\hline 17-OCTADECEN-14-YN-1-OL & & & & 7.37 & \\
\hline 9,12-Octadecadienoic acid (Z,Z)-, ethyl ester & & & 10.88 & & 13.53 \\
\hline (R)-(-)-14-Methyl-8-hexadecyn-1-ol & & & & 0.99 & \\
\hline Ethyl Linoleate & 2.12 & 10.48 & & 10.23 & \\
\hline Hexadecanamide & 1.62 & 1.36 & & 1.96 & \\
\hline lauramide & & & 1.72 & & \\
\hline
\end{tabular}


Table S3. Typical functional groups in the raw sample and solid products.

\begin{tabular}{lll}
\hline No. & Wavenumber $\left(\mathrm{cm}^{-1}\right)$ & Functional group \\
\hline 1 & $3700-3100$ & $-\mathrm{OH}$ and $\mathrm{N}-\mathrm{H}$ stretching vibrations \\
2 & $3000-2840$ & $-\mathrm{CH}$ asymmetrical and symmetrical stretching \\
& & vibrations \\
3 & 1720 & $\mathrm{C}=\mathrm{O}$ stretching vibration in the ester \\
4 & 1709 & $\mathrm{C}=\mathrm{O}$ stretching vibrations of carboxyl \\
5 & 1662 & $\mathrm{C}=\mathrm{O}$ stretching vibrations of aldehyde \\
6 & 1530 & $-\mathrm{NH}$ bending vibration \\
7 & 1234 & $\mathrm{C}-\mathrm{O}$ stretching vibration \\
8 & $1043-1036$ & $\mathrm{C}-\mathrm{O}-\mathrm{C} \quad$ stretching vibration \\
& & polysaccharides \\
9 & 1105 & $\mathrm{C}-\mathrm{O}-\mathrm{C}$ symmetrical stretching vibration, oxygen \\
10 & 918 & heterocyclic ring \\
& & C-O-C asymmetrical stretching vibration, oxygen \\
& & heterocyclic ring
\end{tabular}

